\title{
Author Index Vol. 25, 1996
}

The authors of the abstracts of the Scientific Program 20th Annual Meeting of the American Society of Pediatric Neurosurgery, January 26-February 1, 1997, St. Louis, Mo., are indexed separately in Vol. 25, No. 4, p. 209, 1997.

Abbott,|R. 41 Albright, A.L. 78,93,109 Altmel, Z. 94 Armonda, R. 116 Aronyk, K.E. 210 Ater, J. 174 Aubert, V. 36

Barlas, O. 94 Barnes,|P.D. 252 Barrett,|R. 123 Bayindir, C.. 94 Bejjani, G.K. 269, 302 Bhargava, R. 182 Bissonette,|D.J. 109 Boydston, W.R. 315 Boyett, J. 182 Brockmeyer, D. 325 Broemeling, L.D. 302 Bullock,|M.R. 265 Buoncompagni, A. 323

Camassa, N. 323 Catala, M. 36 Christian, C. 116 Cogen, P.H. 269 Cohen,|A.R. 156 Couanet, D. 7 Curtis,|M. 123

Das, B.S. 248 Davis, R. 174 Day, R.A. 256 Dias, L. 295 Dias, M.S. 210 Donahue, D.J. 269, 302 Doz, F. 7 Drake, J.M. 277 Dufour, Cl 323 Duhaime, A.-Ch. 116,123 Duke, B.J. 31 , 188

Edwards, M.S.B. 174 Epstein, F.J. 41

Faillace, W.J. 321 Farmer,|J.-P. 286 Fewel, M.E. 165 Flickinger, J.C. 109 Frim, D.M. 137 Fujii, M. 227 Fulmer, B.B. 214

Gabrieli, A.P. 295 Gajjar, A. 182

Garrè, M.L. 323 Gaskill, SJJ. 143,147 Gattorno, M. 323 Gennarelli, L.M. 123 George, T.M. 130 Gịlreath, C.L. 315 Gluckman, T.J. 130 Golden,|J. 137 Gooch, J.L. 28 Grabb, P.A. 214 Grant, J.A. 227 Grill,JJ 7

Hadfield, M.G. 100 Hall, Ch.M. 20 Handler, M.H. 276 Harpey, J.-P. 36 Hartmann, O. 7 Hayward, R.D. 20 Hedge,|T. 248 Heideman, R. 182 Heinz,|E.R. 147 Heron,|D. 36 Hertle, R. 116 Hoffman,|H.J. 277 Hsiang, J.N.K. 105 Hudgins, R.J. 315 Humphreys, R.P. 277,326 Hunter,J. 116

izgi, N. 94

Jamjoom, A. 160 Jamjoom, Z.A.B. 160 Jenkins, J.J. 182 Johnson,|D.L. 309 Jones,|B.M. 20 Jones, R. 57

Kail, Y. 54 Kalifa, C. 7 Kandt, R. 147 Kaufman,|B.A. 221,256 Kelly,|D.R. 214 Kestle, J.R. 233 Ketch,|L.L. 31 Kondzịlolka, D. 109 Krishnamurthy, S. 309 Kubal, W. 265 Kun, L.E. 182 Kuratsu, J. 240

Lamborn, K. 174 Langston, J.W. 182 Lesourd, S. 36

Levin,VV.A. 174 Levy, M.L. 165 Li, Y. 182 Lunsford, L.D. 109 Luo, V. 265 Luo, V.Y. 100

McComb, J.G. 165 McLone, D.G. 130,227,295 McPhillips, M. 286 Mapstone, T.B. 93 Marlin, A.E. 143 Martínez-Lage, J.F. 260 Martinez-Perez, D. 252 Martinez Robledo, A. 260 Miller,D.C. 64 Mohanty, A. 248 Molinari, A.C. 323 Montes, J.L. 286 Mori, K 227 Mouchantat, R.A. 31 Muhlbauer, M.S. 1,182 Mulliken, J.B. 252

Nagahịro, S. 54 Naim-Ur-Rahman 160 Nelson,|K.R. 13 Ng, H.-K. 105

Oakes, W.J. 147 O’Gorman,|A.M. 286 Önal, Ç. 94

Packer,|R. 269 Palmer,|C. 214 Park, T.S. 221,256 Partington, M.D. 188 Piatt,|J.H.,JJr. 73 Picco, P. 323 Poon, W.-S. 105 Pople, İ.K》1 Poza, M. 260 Prados, M.D. 174

Rabbit,J. 174 Rekate, H.L. 220 Ritter, A.M. 265 Rooney, C. 156 Rothman, M. 64 Rusin, J. 302 Russo, C.P. 100 Rutka, J.T. 277

Sainte-Rose, C. 7 Sanford, R.A. 1, 182

Sarwark, J.F. 295 Sastry Kolluri, V.R. 248 Schoepfer, C. 7 Scott,|R.M. 137,252 Selby, D. 269 Shaver, E.G. 123 Shaw, D. 20 Sherburn, E.W. 256 Shiminnski-Maher, T. 41 Shultz, B.L. 78 Sirraneci, R. 94 Slaney, S.F. 20 Sola, J. 260 Somaza, S.C. 109 Steinbok, P. 164,233

Tantuwaya, V.S. 221 Tekkök, İ.H. 151 Teo, C, 57

Terrier-Lacombe, M.J. 7 Thompson, D.N.P. 20 Tomita, T. 227 Towbin, R.B. 78 Tsang, R.KY. 105

Ushịo, Y. 54,240

Valteau-Couanet, D. 7 Vander|Woude, D.L. 252 Vauthier-Brouzes, D. 36 Ventureyra,|E.C.G. 151 Vernet, O. 286

Walker,|M.L. 28 Walter, A. 182 Wara, W. 174 Ward, J.D. 100 Warf, B.C, 13 Weber,|D.T. 295 Weịner, H.L. 64 Williams, R.L. 100 Winston,|K.R. 31

Yalçin, I. 94 Yoshioka, S. 54 Yundt, KD. 221

Zec, N. 137 Ziff, E.B. 64 Zimmerman, R.A. 45, 83

\section{KAIIGEH}

() 1996 S. Karger AG, Basel

327

E-Mail karger@karger.ch Fax +41 613061234 http:// www. karger. ch 